VOL. 65 (2002) [55-57]

\title{
FUNCTIONS INVARIANT UNDER THE BOCHNER-MARTINELLI INTEGRAL
}

\section{JAESUNG LEE}

We give an elementary proof of the statement that a function $f$ on the closed unit ball of $\mathbf{C}^{n}$, integrable on the unit sphere, is holomorphic if it is invariant under the Bochner-Martinelli integral transform.

The classical Bochner-Martinelli integral formula can be written explicitly in the unit ball $B_{n}$ of $\mathbf{C}^{n}$. Indeed (see, for example, $[2,16.5 .8]$ ) if $f \in C^{1}\left(\overline{B_{n}}\right)$ and $z \in B_{n}$, then

$$
f(z)=\int_{S} \frac{1-\langle\xi, z\rangle}{|\xi-z|^{2 n}} f(\xi) d \sigma(\xi)-\frac{1}{n} \sum_{k=1}^{n} \int_{B_{n}} \frac{\bar{D}_{k} f(w)\left(\bar{w}_{k}-\bar{z}_{k}\right)}{|w-z|^{2 n}} d \nu(w)
$$

where $S$ is the unit sphere, $\sigma$ is the rotation invariant, positive Borel measure on $S$ with $\sigma(S)=1$ and $\langle\xi, z\rangle=\sum \xi_{k} \bar{z}_{k}$ denotes the inner product in $\mathbf{C}^{n}$ as we follow standard notations of [2]. From the formula (1), we see that if $f$ is, in addition, holomorphic in $B_{n}$ then for $z \in B_{n}$

$$
f(z)=\int_{S} \frac{1-\langle\xi, z\rangle}{|\xi-z|^{2 n}} f(\xi) d \sigma(\xi) .
$$

Now, for $f \in L^{1}(S)$, we define an integral transform $B f$ on $B_{n}$ by

$$
(B f)(z)=\int_{S} \frac{1-\langle\xi, z\rangle}{|\xi-z|^{2 n}} f(\xi) d \sigma(\xi) \quad \text { for } z \in B_{n}
$$

We call $B f$ the Bochner-Martinelli transform of $f$. For $n=1, B f$ is the Cauchy integral (thus it is holomorphic), but in general not holomorphic when $n \geqslant 2$ although it is harmonic in $B_{n}$ for every $f \in L^{\mathbf{1}}(S)$.

In 1978 Romanov [1] showed, by using the iterates of the operator $B$, that a function $f \in C\left(B_{n}\right) \cap L^{2}(S)$ satisfies $B f=f$ on $B_{n}$, then it is holomorphic. Here we prove, by an elementary method, a function on the closed unit ball, integrable on $S$, which is invariant under the Bochner-Martinelli transform is holomorphic.

TheOREM 1. If a function $f$ on $\overline{B_{n}}$ which is integrable on $S$ satisfies

$$
f(z)=(B f)(z) \quad \text { for all } z \in B_{n}
$$

then $f$ is holomorphic in $B_{n}$.

Received 26th April, 2001

Copyright Clearance Centre, Inc. Serial-fee code: 0004-9727/02 \$A2.00+0.00. 
PROOF: It is trivial for $n=1$, thus we assume that $n \geqslant 2$. Since $f$ is harmonic in $B_{n}$ by $(3), f(z)=P f(z)$ for all $z \in B_{n}$ where

$$
(P f)(z)=\int_{S} \frac{1-|z|^{2}}{|\xi-z|^{2 n}} f(\xi) d \sigma(\xi)
$$

is the Poisson integral of $f$. Thus for each $z \in B_{n}$, we have

$$
\begin{aligned}
0 & =P f(z)-B f(z) \\
& =\int_{S} \frac{\langle\xi, z\rangle-|z|^{2}}{|\xi-z|^{2 n}} f(\xi) d \sigma(\xi) \\
& =\sum_{k} \bar{z}_{k} \int_{S} \frac{\xi_{k}-z_{k}}{|\xi-z|^{2 n}} f(\xi) d \sigma(\xi) \\
& =\sum_{k} \bar{z}_{k} \frac{\partial}{\partial \bar{z}_{k}} h(z)
\end{aligned}
$$

where

$$
h(z)=\frac{1}{n-1} \int_{S} \frac{f(\xi)}{|\xi-z|^{2 n-2}} d \sigma(\xi)
$$

(it is the single layer potential with movement $f$ ).

Since $h$ is real analytic (indeed it is harmonic) in $B_{n}$, the power series expression of $h$ at the origin shows that (4) implies that $h$ is holomorphic.

Hence the function

$$
g(z)=\sum z_{k} \frac{\partial}{\partial z_{k}} h(z)
$$

is also holomorphic.

Once again by the same calculation as (4), we can see that

$$
g(z)=\int_{S} \frac{\langle z, \xi\rangle-|z|^{2}}{|\xi-z|^{2 n}} f(\xi) d \sigma(\xi)
$$

Therefore

$$
\begin{aligned}
B f(z) & =\int_{S} \frac{1-\langle\xi, z\rangle}{|\xi-z|^{2 n}} f(\xi) d \sigma(\xi) \\
& =\int_{S}\left(\frac{\langle z, \xi\rangle-|z|^{2}}{|\xi-z|^{2 n}}+\frac{|\xi-z|^{2}}{|\xi-z|^{2 n}}\right) f(\xi) d \sigma(\xi) \\
& =g(z)+(n-1) h(z)
\end{aligned}
$$


The holomorphicity of $g$ and $h$ implies that $B f$ is holomorphic.

Since $f=B f, f$ is also holomorphic and this proves the theorem.

\section{REFERENCES}

[1] A. Romanov, 'Spectral analysis of the Martinelli-Bochner operator for the ball in $\mathrm{C}^{n}$ and its application', Funct. Anal. Appl. 12 (1978), 232-234.

[2] W. Rudin, Function theory in the unit ball of $\mathbf{C}^{n}$ (Springer-Verlag, Berlin, Heidelberg, New York, 1980).

Department of Mathematics

Sogang University

Seoul 121-742

Korea

e-mail: jalee@sogang.ac.kr 